\title{
Design of a $\times 4$ subharmonic sub-millimeter wave diode mixer, based on an analytic expression for small-signal conversion admittance parameters
}

\author{
Michaelsen, Rasmus Schandorph; Johansen, Tom Keinicke; Krozer, Viktor
}

Published in:

2013 Microwave \& Optoelectronics Conference (IMOC)

Link to article, DOI:

10.1109/IMOC.2013.6646457

Publication date:

2013

Link back to DTU Orbit

Citation (APA):

Michaelsen, R. S., Johansen, T. K., \& Krozer, V. (2013). Design of a $\times 4$ subharmonic sub-millimeter wave diode mixer, based on an analytic expression for small-signal conversion admittance parameters. In 2013 Microwave \& Optoelectronics Conference (IMOC) IEEE. https://doi.org/10.1109/IMOC.2013.6646457

\section{General rights}

Copyright and moral rights for the publications made accessible in the public portal are retained by the authors and/or other copyright owners and it is a condition of accessing publications that users recognise and abide by the legal requirements associated with these rights.

- Users may download and print one copy of any publication from the public portal for the purpose of private study or research.

- You may not further distribute the material or use it for any profit-making activity or commercial gain

- You may freely distribute the URL identifying the publication in the public portal 


\title{
Design of a $\times 4$ subharmonic sub-millimeter wave diode mixer, based on an analytic expression for small-signal conversion admittance parameters
}

\author{
Rasmus S. Michaelsen ${ }^{1}$, Tom K. Johansen ${ }^{1}$ and Viktor Krozer ${ }^{2}$ \\ ${ }^{1}$ Technical University of Denmark, Department of Electrical Engineering \\ 2800 Kongens Lyngby, Denmark \\ Email: rsmi@elektro.dtu.dk \\ ${ }^{2}$ Goethe-Universitt Frankfurt am Main, Physikalisches Institut \\ D-60438 Frankfurt am Main, Germany
}

\begin{abstract}
Instead of using frequency multipliers before a fundamental mixer, subharmonic mixers can be used. In order to develop novel subharmonic mixer architectures it is necessary to know the exact signal phase at the nonlinear element. The purpose of this paper is to generalize the description of the smallsignal admittance in a Schottky-diode mixer where the phase can be set arbitrarily. It is shown that only for the case of a fundamental frequency mixer this admittance becomes a purely real valued conductance.

To test the theory a $\times 4$ subharmonic sub-millimeter wave mixer is designed and simulated. With an RF frequency of 640 $\mathrm{GHz}$, this design achieves a conversion gain of $-13.5 \mathrm{~dB}$ with a LO-power of only $-2.5 \mathrm{dBm}$.
\end{abstract}

Index Terms-Mixer, sub-harmonic, sub-millimeter wave.

\section{INTRODUCTION}

For millimeter- and sub-millimeter- wave bands it is common practice to use multiplier chains to obtain the necessary local oscillator (LO) frequency from a reliable voltage controlled oscillator (VCO) [1]. Sub-harmonic mixers utilize the higher harmonics of the LO signal such that a lower frequency from the VCO is required, thus eliminating requirements for multiplier steps. In [2] a structure for a $\times 4$ subharmonically pumped resistive HEMT has been suggested. The idea is to have the nonlinear elements driven $90^{\circ}$ out of phase. This is also possible for Schottky-diode based mixers. It is known that this phase condition leads to cancellation of second and third harmonic signals. However, a general theoretical description of mixer performance with arbitrary phase is still missing, but is important in order to understand the impact of phase variations.

The purpose of this paper is to generalize the description of the small-signal conversion admittance in a diode mixer where the phase can be set arbitrarily. It is shown that only for the case of a fundamental frequency mixer this admittance becomes a purely real valued conductance. Knowing the admittance of the Schottky diodes verifies the possibility of design of the above described circuit and makes novel subharmonic mixer topologies possible.

This theory has been verified by design and simulation of a $\times 4$ subharmonic mixer operating with an RF-frequency of

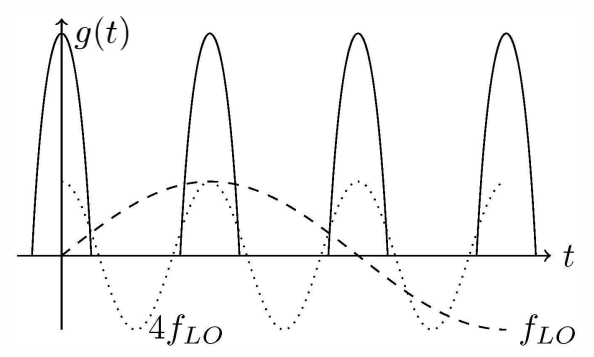

Fig. 1. The conductance waveform of the $\times 4$ subharmonically pumped resistive mixer

$640 \mathrm{GHz}$. The mixer behaves well and the performance is comparable to state-of-the-art subharmonic mixers.

\section{THEORY}

The novel idea of [2] can be used for any resistive mixer. The idea is to drive four nonlinear resistive elements with consecutive increasing $90^{\circ}$ phase shift. This gives a conductance waveform of the resistive element similar to that of a single element driven with a four times higher LO, see figure 1. The purpose of this approach is to reuse the power at the idle frequencies by phase cancellation instead of filtering. This could theoretically be extended to any number of higher-order subharmonic mixers, somewhat similar to what is proposed by [3].

Using the harmonic balance and conversion matrix theory of [4], this requires the fourth Fourier component of the diode conductances to sum constructively and cancel for the lower harmonics. The conversion matrix theory can also be used to analyze the circuit if the Fourier components of the conductance is known.

The I-V characteristic for a Schottky-Diode is

$$
I(V)=I_{s}\left(e^{\frac{V}{N_{f} V_{t}}}-1\right)
$$

where $I_{s}$ is the saturation current, $N_{f}$ is the ideality factor and $V_{t}$ is the thermal voltage. The small-signal conductance 
waveform of a Schottky-diode is then [4]

$$
g(t)=\left.\frac{\mathrm{d} I(V)}{\mathrm{d} V}\right|_{V=V_{L}(t)}=\frac{I_{s}}{N_{f} V_{t}} e^{\frac{V_{L}(t)}{N_{f} V_{t}}}
$$

where $V_{L}(t)=V_{D C}+V_{L o} \cos \left(\omega_{0} t+\phi\right)$ is the large-signal voltage.

This conductance is periodic and can be described as a Fourier series where the coefficients can be found as

$$
\begin{aligned}
Y_{n}= & \frac{I_{s}}{N_{f} V_{t}} e^{\frac{V_{D C}}{N_{f} V_{t}}} \frac{1}{T} \int_{0}^{T} e^{\frac{V_{L} \operatorname{c\bullet s}\left(\omega_{\bullet} t+\phi\right)}{N_{f} V_{t}}} e^{-j n \omega_{\bullet} t} \mathrm{~d} t \\
= & A_{0} \frac{1}{2 \pi} \int_{-\pi}^{\pi} e^{x \cos \left(\omega_{\bullet} t+\phi\right)} e^{-j n \omega_{\bullet} t} \mathrm{~d} \omega_{0} t \\
& \text { where } A_{0}=\frac{I_{s}}{N_{f} V_{t}} e^{\frac{V_{D C}}{N_{f} V_{t}}} \text { and } x=\frac{V_{L o}}{N_{f} V_{t}}
\end{aligned}
$$

Multiplying with $1=e^{-j n \phi} e^{j n \phi}$ gives

$$
\begin{aligned}
Y_{n} & =A_{0} \frac{1}{2 \pi} \int_{-\pi}^{\pi} e^{x \cos \left(\omega_{\bullet} t+\phi\right)} e^{-j n \omega_{\bullet} t} e^{-j n \phi} e^{j n \phi} \mathrm{d} \omega_{0} t \\
& =A_{0} e^{j n \phi} I_{n}(x) \text { for } n \in \mathbb{Z}
\end{aligned}
$$

where $I_{n}(x)$ is the modified Bessel-function of order $n$ and $\operatorname{argument} x$. This shows that the phase of the Fourier coefficients changes with the phase of the LO times the harmonic number.

The $\times 4$ subharmonic mixer in figure 2 will have Fourier coefficients given as (5) in the bottom of this page. As expected they cancel for all harmonics not divisible by four and adds constructively for the rest. It is interesting to see that with this theoretical background one can immediately construct a $\times 3$ subharmonic mixer with LO signal angles equal to 0deg, 120deg, and $240 \mathrm{deg}$, respectively. Also in this case all contributions cancels apart from the ones at DC and the third harmonic. This shows the usefulness of the presented generalization.

\section{DESIGN}

In this section the previously described theory is tested, by design and simulation of $\mathrm{a} \times 4$ subharmonic mixer with an RF frequency of $640 \mathrm{GHz}, \mathrm{LO}$ frequency of $159,5 \mathrm{GHz}$ and IF frequency of $2 \mathrm{GHz}$, in Agilents Advanced Design System (ADS).

The SD1T7-D20 diode from [5] is used as the resistive element. The diode data used for the ADS model is

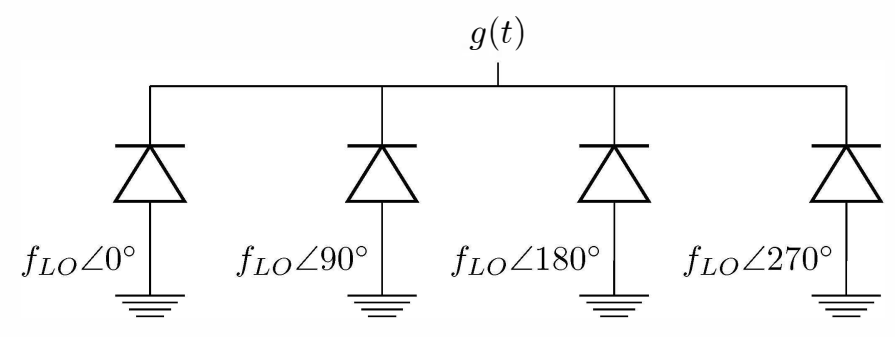

Fig. 2. Four diode $\times 4$ subharmonic mixer, driven by LO signal with $90^{\circ}$ consecutive phase propagation.

- Saturation current, $I_{s}=2 \cdot 10^{-16} \mathrm{~A}$

- Series resistance, $R_{s}=14 \Omega$

- Ideality factor, $n=1.3$

- Zero junction capacitance, $C_{j o}=1.3 f F$

- Parasitic capacitance for one anti-parallel diode pair, $C_{p}=5 f F$

The optimal impedance, for a low LO drive level, seen from the diodes is found to be

- $Z_{I F}=439-j 23 \Omega$

- $Z_{L O}=15.6-j 140 \Omega$

- $Z_{R F}=3.5-j 31 \Omega$

In figure 3 is the diagram of the circuit shown. The $90 \mathrm{deg}$ consecutive phase propagation of the LO drive is made by using two anti parallel diode pairs and having the LO filter implement a 90 deg hybrid. Both the RF- and LO- filter and matching is realized using microstrip design.

Due to the high frequencies the substrate has to be so thin that it is not possible to have longitudinal waves. To make this substrate, a thin layer of dielectric can be deposited on the ground plane. The substrate parameters used for the microstrip line implementation and simulation is:

- Height, $h=6 \mu m$

- Relative permittivity, $\epsilon_{r}=3.6$

- Relative permeability, $\mu_{r}=1$

- Loss tangent, $\tan \delta=10^{-3}$

\section{Simulation Results}

The $\times 4$ sumharmonic mixer is simulated using harmonic balance simulations in ADS. The conversion gain of the mixer is found to be $G_{c n v}=-13.2 d B$ at a low LO power level of only $P_{L O}=-2.5 \mathrm{dBm}$. Table I compares this design to other state-of-the-art mixers. Note that this $\times 4$ subharmonic

$$
\begin{gathered}
f_{L O} \angle 0^{\circ} \quad f_{L O} \angle 90^{\circ} \quad f_{L O} \angle 180^{\circ} \quad f_{L O} \angle 270^{\circ} \\
G_{0}=A_{0} I_{0}(x) e^{j 0 \cdot 0}+A_{0} I_{0}(x) e^{j 0 \cdot \frac{1}{2} \pi}+A_{0} I_{0}(x) e^{j 0 \cdot \pi}+A_{0} I_{0}(x) e^{j 0 \cdot \frac{3}{2} \pi}=4 A_{0} I_{0}(x) \\
G_{1}=A_{0} I_{1}(x) e^{j 1 \cdot 0}+A_{0} I_{1}(x) e^{j 1 \cdot \frac{1}{2} \pi}+A_{0} I_{1}(x) e^{j 1 \cdot \pi}+A_{0} I_{1}(x) e^{j 1 \cdot \frac{3}{2} \pi}=0 \\
G_{2}=A_{0} I_{2}(x) e^{j \cdot 0}+A_{0} I_{2}(x) e^{j 2 \cdot \frac{1}{2} \pi}+A_{0} I_{2}(x) e^{j 2 \cdot \pi}+A_{0} I_{2}(x) e^{j 2 \cdot \frac{3}{2} \pi}=0 \\
G_{3}=A_{0} I_{3}(x) e^{j 3 \cdot 0}+A_{0} I_{3}(x) e^{j 3 \cdot \frac{1}{2} \pi}+A_{0} I_{3}(x) e^{j 3 \cdot \pi}+A_{0} I_{3}(x) e^{j 3 \cdot \frac{3}{2} \pi}=0 \\
G_{4}=A_{0} I_{4}(x) e^{j 4 \cdot 0}+A_{0} I_{4}(x) e^{j 4 \cdot \frac{1}{2} \pi}+A_{0} I_{4}(x) e^{j 4 \cdot \pi}+A_{0} I_{4}(x) e^{j 4 \cdot \frac{3}{2} \pi}=4 A_{0} I_{4}(x)
\end{gathered}
$$




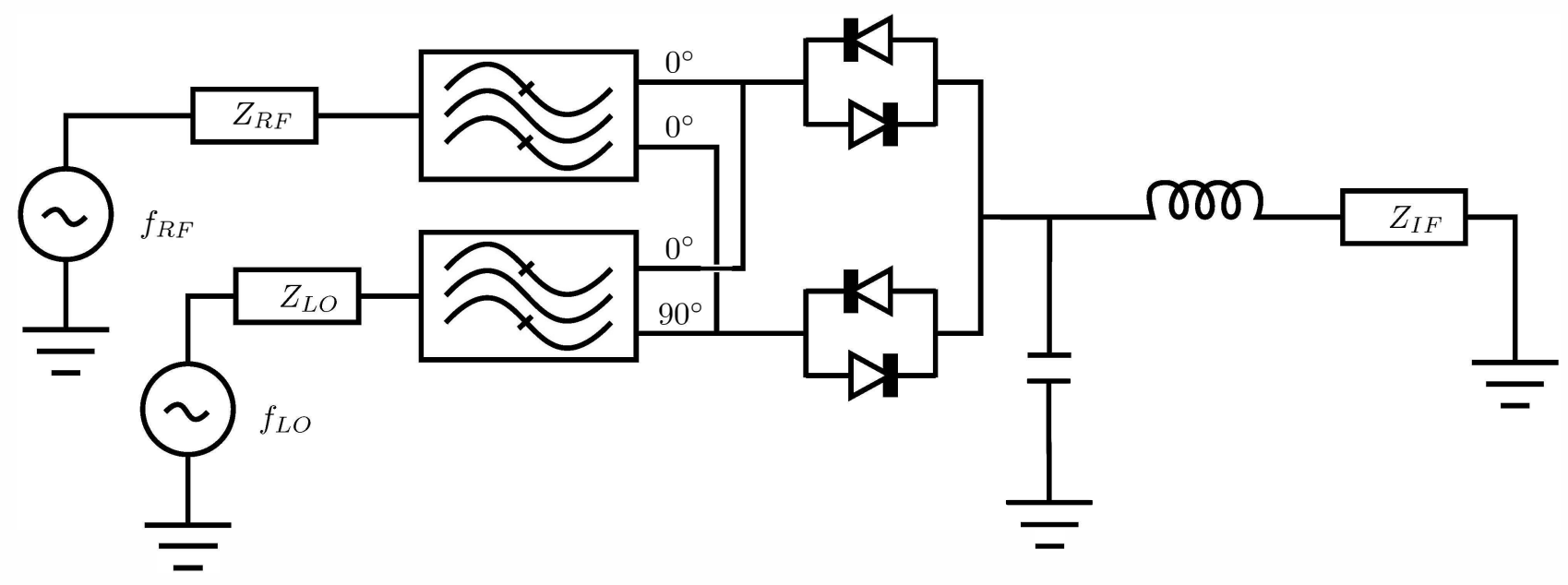

Fig. 3. Diagram of circuit for the $\times 4$ subharmonic mixer

TABLE I

STATE-OF-THE-ART MIXERS FOUND IN THE LITERATURE

\begin{tabular}{crlrrrr}
\hline Source & Rf frequency & Type & IRR & Lo Power & Bias [mV] & $G_{C}[\mathrm{~dB}]$ \\
\hline$[6]$ & $21 \mathrm{GHz}-40 \mathrm{GHz}$ & $2 \times$ SH HEMT & - & $10.5 \mathrm{dBm}$ & None & -8.2 to -14.3 \\
{$[7]$} & $182 \mathrm{GHz}$ & $2 \times$ SH Diode & - & $6.5 \mathrm{dBm} / 9.5 \mathrm{dBm}$ & $400 \mu A / 0$ & -12.7 \\
{$[8]$} & $170 \mathrm{GHz}-210 \mathrm{GHz}$ & $2 \times$ SH Diode & - & $10 \mathrm{dBm}$ & $1.1 \mathrm{~mA}$ & -16 to -12 \\
{$[9]$} & $390 \mathrm{GHz}$ & $2 \times$ SH Diode & - & Not reported & None & -13.9 \\
{$[10]$} & $640 \mathrm{GHz}$ & $2 \times$ SH Diode & - & $<5.3 \mathrm{dBm}$ & None & -12 \\
{$[11]$} & $800 \mathrm{GHz}-856 \mathrm{GHz}$ & $2 \times$ SH Diode & - & Not reported & Not reported & -15 to -13.5 \\
{$[12]$} & $180 \mathrm{GHz}-196 \mathrm{GHz}$ & $4 \times$ SH Diode & $>15 \mathrm{~dB}$ & 18.7 & None & -30 to -20 \\
{$[2]$} & $60 \mathrm{GHz}$ & $4 \times$ SH HEMT & - & $8 \mathrm{dBm}$ & None & -15.3 \\
This work & $640 \mathrm{GHz}$ & $4 \times$ SH Diode & - & $-2.5 \mathrm{dBm}$ & None & -13.2 \\
\hline
\end{tabular}

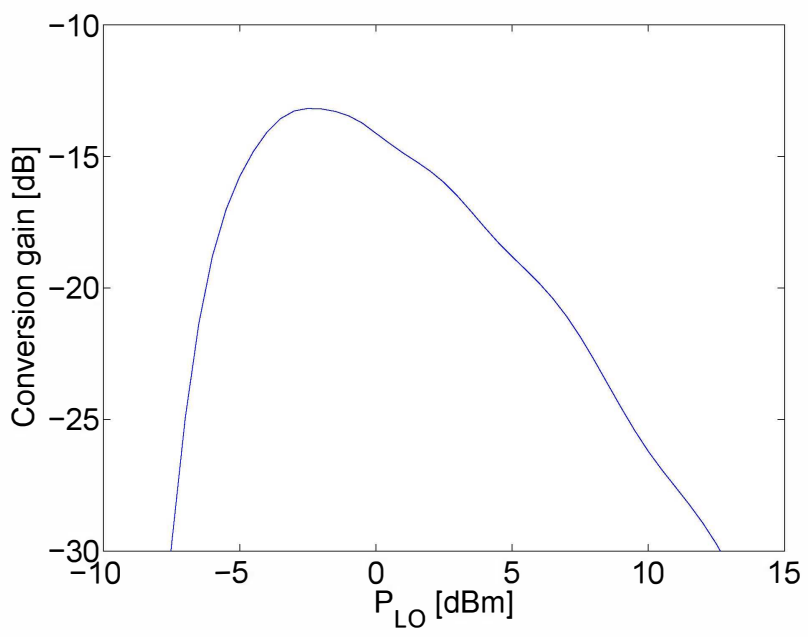

Fig. 4. Conversion gain plotted versus LO power for the $\times 4$ subharmonic mixer using microstrip lines.

mixer, achieves good conversion with less power than any of the compared. This is very important as signal generation at sub-millimeter wave lengths, is troublesome and includes different sets of amplifiers and frequency doublers. In figure 4 the conversion gain is plotted versus available LO power. As can be seen the conversion gain is above $-15 d B$ for LO power levels from $-4.5 \mathrm{dBm}$ to $1 \mathrm{dBm}$.

\section{CONCLUSION}

It was shown, that the Fourier elements of the admittance for a Schottky-diode can be evaluated as $G_{n}=A_{0} e^{j n \phi} I_{n}(x)$, which becomes complex in contrast to fundamental frequency mixers. The phase of the admittance is equal to the phase of the LO signal times the harmonic. This implies that to make a circuit where the Fourier components cancel up to the desired harmonic, $m$, where they interact constructively, one should choose the phase progression $\phi=\frac{360^{\circ}}{m}$.

To test the theory a $640 \mathrm{GHz} \times 4$ subharmonic mixer was designed and simulated. The design utilized two pair of antiparallel diodes which was connected through $90^{\circ}$ Hybrid and filters for LO and a power divider and filter for RF. Both filters include matching. The conversion gain of the designed mixer is $-13.2 \mathrm{~dB}$ with a low LO power of only $-2.5 \mathrm{dBm}$.

\section{REFERENCES}

[1] V. Krozer, G. Loata, J. Grajal de la Fuente, and P. Sanz, "Limitations in $\mathrm{THz}$ power generation with Schottky diode varactor frequency multipliers," Proceedings, IEEE Tenth International Conference on Terahertz Electronics, pp. 109-112, 2002.

[2] S. Gunnarsson, "Analysis and Design of a Novel X4 Subharmonically Pumped Resistive HEMT Mixer," IEEE Transactions on Microwave Theory and Techniques, vol. 56, no. 4, pp. 809-816, 2008.

[3] C. Yao and J. Xu, "A novel circuit architecture for high performance of high-order subharmonic mixers," International Journal of Infrared and Millimeter Waves, vol. 29, no. 8, pp. 748-757, 2008

[4] S. A. Maas, Nonlinear Microwave and RF Circuits, 2nd ed. Artech House, Inc., 1988. 
[5] B. Thomas, A. Maestrini, and G. Beaudin, "A low-noise fixed-tuned 300-360-GHz sub-harmonic mixer using planar Schottky diodes," IEEE Microwave and Wireless Components Letters, vol. 15, no. 12, pp. 865867, 2005.

[6] S.-H. Hung, Y.-C. Lee, C.-C. Su, and Y.-H. Wang, "High-Isolation Millimeter-Wave Subharmonic Monolithic Mixer With Modified QuasiCirculator," IEEE Transactions on Microwave Theory and Techniques, vol. 61, no. 3, pp. 1140-1149, 2013.

[7] T.-H. Lee, C.-Y. Chi, J. East, G. Rebeiz, and G. Haddad, "A quasioptical subharmonically-pumped receiver using separately biased Schottky diode pairs," 1994 IEEE MTT-S International Microwave Symposium Digest (Cat. No.94CH3389-4), pp. 783-786 vol.2, 1994.

[8] M. Morgan, "Millimeter-Wave MMICs and Applications," Ph.D. dissertation, California Institute of Technology, 2003.
[9] J. Treuttel, B. Thomas, A. Maestrini, H. Wang, B. Aldernan, J. Siles, S. Davis, and T. Narhi, "A $380 \mathrm{GHz}$ sub-harmonic mixer using MMIC foundry based Schottky diodes transferred opnto quartz substrate, 20th International Symposium on Space Terahertz Technoogy, pp. 251-254, 2009.

[10] I. Mehdi, P. Siegel, D. Humphrey, T. Lee, R. Dengler, J. Oswald, A. Pease, R. Lin, H. Eisele, R. Zimmermann, and N. Erickson, "An all solid-state $640 \mathrm{GHz}$ subharmonic mixer," 1998 IEEE MTT-S International Microwave Symposium Digest (Cat. No.98CH36192), vol. 2 , pp. 403-406 vol.2, 1998

[11] B. Thomas, A. Maestrini, D. Matheson, I. Mehdi, and P. de Maagt, "Design of an $874 \mathrm{GHz}$ biasable sub-harmonic mixer based on MMIC membrane planar schottky diodes," 2008 33rd International Conference on Infrared, Millimeter and Terahertz Waves, pp. 1-2, 2008.

[12] J. W. Archer and J. Tello, "A 180-196 GHz image-reject schottky-diode MMIC mixer," Microwave and optical technology letters, pp. 23192322, 2007. 